\title{
Severity Two Out of Four
}

National Cancer Institute

\section{Source}

National Cancer Institute. Severity Two Out of Four. NCI Thesaurus. Code C147503.

A severity result of two, on a four level scale. 